\title{
An Analysis of Transient Flow in a Clean Room by a Modified GSMAC Finite Element Method*
}

\author{
Keisuke SUESADA**, Mitsuhiko TAKEMURA***, \\ Takahiko TANAHASHI ${ }^{* * *}$ and Eiji KASAHARA*****
}

\begin{abstract}
Clean room facilities have recently become indispensable to various kinds of industries. In order to design a clean room, it is important to consider the improvement of the working environment as well as energy conservation. The air flow in a clean room is very complex. It consists of recirculation flows, and the direction of mean air flow is changed locally. A method of numerical simulation has been developed to delineate guidelines for the ventilation design of a clean room. This paper presents a modified finite element analysis for solving unsteady incompressible Navier-Stokes equations. This scheme is based on the algorithm of the HSMAC (highly simplified marker and cell) method in SOLA and the general idea of orthogonal decomposition. It has actually been applied to the case of the lid-driven square cavity flow at Reynolds number 10000 , and to air flow in a conventional clean room. Unsteady flow simulations in terms of stream lines, equipressure lines and equivorticity lines are obtained on a color graphic display.
\end{abstract}

Key Words: Fluid Dynamics, Computational Fluid Dynamics, Finite Element Method, Clean Room, Fluid Transients, Flow Visualization

\section{Introduction}

For the maximum removal of dust in air, clean rooms have recently become indispensable to various kinds of industries: e.g., electronics, fine machinery and photograph. In the form of a biological clean room, application fields are extending to the medical and pharmaceutical industries and even to such surgical operations on the brain and heart. We can say that the clean room gives us an indispensable working environment. In such a clean room, the dust in air is removed and diffused by air streams, and it is there-

* Received 21st February, 1990. Paper No. 88-1394B

** Chiyoda Corporation, 37-37 Tsurumichuo 4-chome, Tsurumi-ku, Yokohama 230, Japan

*** Department of Mechanical Engineering, Keio University, 14-1, Hiyoshi 3-chome, Kohoku-ku, Yokohama 223, Japan

**** Research and Development, Takasago Thermal Engineering Co., Ltd., 3150 Iiyama, Atsugi 243-02, Japan

*****Department of Mechanical Engineering, Musashi Institute of Technology, 28-1, Tamazutsumi 1chome, Setagaya-ku, Tokyo 158, Japan fore very important to succinctly control the distribution of air streams in the room. As a contribution to the purification of air, air stream behavior in a clean room attracts much attention.

In this field of research, until now, there have been many experimental studies, but as yet, not many computational examples. In this study, we present a new scheme ${ }^{(1)}$ which adopts the algorithm of HSMAC (highly simplified marker and cell method) and mathematical concepts of orthogonal decomposition of flow fields. We also perform a numerical simulation which clarifies the transient behavior of flow in a clean room.

\section{Modifled GSMAC Finite Element Method}

The FEM presented here features the extended application of an algorithm developed by the finite difference method to an arbitrary mesh system in FEM and the concept of orthogonal decomposition in a flow field devised by Cholin ${ }^{(2)}$, which is defined as the modified GSMAC method. The outline of this scheme is as follows.

\subsection{Orthogonal decomposition}

The basic equations are as follows: 


$$
\begin{aligned}
& \frac{\partial \vec{u}}{\partial t}=-\nabla H-\frac{1}{R e} \nabla \times \vec{\omega}+u \times \vec{\omega} \\
& \nabla \cdot \vec{u}=0 \\
& \vec{\omega}=\nabla \times \vec{u}, H=p+\frac{u^{2}}{2},
\end{aligned}
$$

where $\vec{\omega}$ is the vorticity vector and $\boldsymbol{H}$ is the Bernoulli function.

For Eq. ( 1 ), by discretizing the velocity explicitly and the pressure implicitly, we obtain

$$
\begin{aligned}
& \frac{\vec{u}^{n+1}-\vec{u}^{n}}{\Delta t}=-\nabla H^{n+1}-\frac{1}{R e} \nabla \times \vec{\omega}^{n}+\vec{u}^{n} \times \vec{\omega}^{n} \\
& \nabla \cdot \vec{u}^{n+1}=0 .
\end{aligned}
$$

Decomposing Eq. (4) in terms of the predicted velocity vector $\tilde{\boldsymbol{u}}^{n+1}$ (orthogonal decomposition), we obtain

$$
\begin{aligned}
& \frac{\tilde{u}^{n+1}-\vec{u}^{n}}{\Delta t}=-\nabla H^{n}-\frac{1}{R e} \nabla \times \vec{\omega}^{n}+\vec{n}^{n} \times \vec{\omega}^{n}(6) \\
& \frac{\vec{u}^{n+1}-\tilde{u}^{n+1}}{\Delta t}=-\nabla H^{*}, H^{*}=H^{n+1}-H^{n} \cdots \\
& \nabla \cdot \vec{u}^{n+1}=0 .
\end{aligned}
$$

Taking the divergence of Eq. $(7)$, we obtain

$\nabla \cdot \vec{u}^{n+1}-\nabla \cdot \tilde{u}^{n+1}=-\Delta t \nabla^{2} H^{*}$.

In the ordinary GSMAC method ${ }^{(3)(4)}$, we obtain $\boldsymbol{H}^{*}$ by solving Poisson's equatlon $\tilde{\nabla} \boldsymbol{u}^{n+1} / \Delta \boldsymbol{t}=\nabla^{2} \boldsymbol{H}^{*}$ in condition $\nabla \overrightarrow{\boldsymbol{u}}^{n+1}$ at the $(n+1)$ th time step; then, we calculate $\overrightarrow{\boldsymbol{u}}^{n+1}$ by using $\boldsymbol{H}^{*}$. While in this scheme, we derive $\boldsymbol{H}^{*}$ by Eq. ( 9 ) and the new prediction of the velocity vector $\tilde{\boldsymbol{u}}^{n+1}$ is derived from Eq. $(7)$, which we substitute into Eq. ( 9 ).

By iterating this calculation, we obtain the correct velocity vector at the $(n+1)$ th time step, and finally $\left(\overrightarrow{\boldsymbol{u}}^{n+1}-\tilde{\boldsymbol{u}}^{n+1}\right)$ and $\boldsymbol{H}^{*}$ become equal to zero.

\section{2 Boundary conditions}

we assume the following two boundary condi. tions, which are given on $\Gamma_{1}$ and $\Gamma_{2}$, a part of the entire boundary $\Gamma$ :

$$
\begin{aligned}
& u=\hat{u}, v=\hat{v} \quad\left(\text { on } \Gamma_{1}\right) \\
& H \cdot l+\frac{\omega}{R e} \cdot m=b_{x} \\
& H \cdot m+\frac{\omega}{R e} \cdot l=b_{y} \quad\left(\text { on } \Gamma_{2}\right),
\end{aligned}
$$

where $\hat{\boldsymbol{u}}, \hat{\boldsymbol{v}}$ are the boundary values and $\boldsymbol{b}_{x}, \boldsymbol{b}_{y}$ the prescribed values on the boundary $\Gamma_{2}$.

The boundary $\Gamma_{1}, \Gamma_{2}$ must satisfy

$$
\begin{aligned}
& \Gamma_{1} \cup \Gamma_{2}=\Gamma \\
& \Gamma_{1} \cap \Gamma_{2}=\phi .
\end{aligned}
$$

\section{3 Correction of $\boldsymbol{H}$ by iteration}

We obtain $\boldsymbol{H}^{*}$ and then the corrected value of $\boldsymbol{H}$ by iterating the procedure of successive approximation. Replacing $\phi=\Delta \boldsymbol{t} \boldsymbol{H}^{*}$ into Eq. ( 7 ), we obtain $\nabla^{2} \phi=\nabla \cdot \tilde{u}^{n+1}$

We multiply the above equation by a weight function, a constant over each element and integrate it; then
$\int_{\Omega e} \nabla^{2} \phi d \Omega=\int_{\Omega e} \nabla \cdot \tilde{u}^{n+1} d \Omega$.

By means of the Gauss divergence theorem, Eq. (15) can be written as

$$
\oint_{\Gamma^{e}}\left(\hat{u} \cdot \vec{n}-\frac{\partial \phi}{\partial n}\right) d \Gamma=0,
$$

where $\boldsymbol{\Gamma}^{\boldsymbol{e}}$ is the boundary of element $\overrightarrow{\boldsymbol{e}}$ and $\boldsymbol{n}$ indicates the outward normal unit vector at a point on the boundary of an element.

In order to facilitate the iteration procedure, we modify Eq. (16) as follows :

$$
\begin{aligned}
& \phi^{k_{2}+1}=\phi^{k_{2}}-\frac{\lambda \Delta \tau}{S^{e}}\left(D^{k}-\oint_{\Gamma^{e}} \frac{\partial \phi^{k_{2}}}{\partial n} d \Gamma\right) \\
& D^{k}=\oint_{\Gamma^{e}} \tilde{u} \cdot \vec{n} d \Gamma,
\end{aligned}
$$

where $\lambda$ is the relaxation factor, and $\boldsymbol{S}^{\boldsymbol{e}}$ expresses an area of element $e$

$$
\begin{aligned}
& \vec{u}^{k+1}=\vec{u}^{k}-\nabla \phi^{k_{2}+1} \\
& H^{k+1}=H^{k}+\frac{\phi^{k_{2}+1}}{\Delta t} .
\end{aligned}
$$

We must repeat the calculation of Eq. (17) starting from $\phi=0$ until the corrected values of velocity and pressure obtained satisfy the following Eq.(21) :

$D^{k}<\varepsilon$.

This method differs from the HSMAC method ${ }^{(5)}$; in order to avoid checkerboard-type pressure oscillation, we replace $u$ and $v$ in the center on the side of the element with the contour integration $\partial \phi / \partial n$. This may make it possible to calculate the exact flux across the side of the element regardless of the shape; therefore, application to FEM may be feasible.

The calculation of contour integration can be performed as follows. When an element $\mathbf{K}$ contacts another one $\mathbf{L}$ with line $\mathbf{a b}, \operatorname{grad} \phi$ in the element KaLb can be calculated by the following equation :

$$
\operatorname{grad} \phi=\frac{1}{S_{a b}} \oint_{\mathrm{KaLb}} \phi \vec{n} d \Gamma,
$$

where $S_{a b}$ is the area of the quadrangle KaLb.

Since the normal vector on side $\mathbf{a b}$ is easily obtained, the line integral is written as follows :

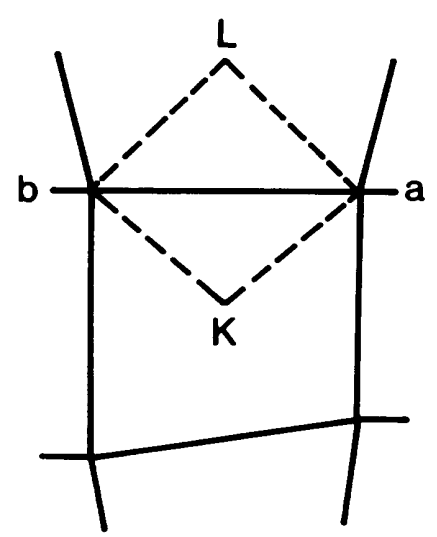

Fig. 1 Closed path for integration 


$$
\begin{aligned}
& \int_{a b} \frac{\partial \phi}{\partial n} d \Gamma=A_{1}\left(\phi_{\mathrm{L}}-\phi_{K}\right)+A_{2}\left(\phi_{b}-\phi_{a}\right) \\
& A_{1}=\frac{l_{a b}^{2}}{2 S_{a b}} \\
& A_{2}=\frac{\overrightarrow{K L} \cdot \overrightarrow{b a}}{2 S_{a b}},
\end{aligned}
$$

where $l_{a b}$ is the length of side ab. Therefore, if $\boldsymbol{A}_{1}$ and $\boldsymbol{A}_{2}$ are obtained previously, the line integral may be calculated in a short time.

The relaxation factor $\lambda$ in Eq. (17), original in this scheme, is a parameter which plays a very important role in the convergence of the solution by successive approximation. The value of $\lambda$ differs according to the problem to be solved and can only be obtained by trial and error. In the case of employing the minimum area element as $\boldsymbol{\Delta} \tau$, a suitable value for $\lambda$ is between $0.1 \sim$ 0.2 .

\section{4 Algorithm of solution}

Procedures of computation are summarized as follows.

Step 1. Given $\overrightarrow{\boldsymbol{u}}^{n}$ and $\boldsymbol{H}^{n}, \tilde{\boldsymbol{u}}^{n+1}$ is calculated from Eq. ( 6 ) ; let it be a prediction value.

Step 2. By iterating the calculation of Eq. (17), $\phi$ is obtained and the velocity of flow $\overrightarrow{\boldsymbol{u}}^{k}$ and the Bernoulli function $\boldsymbol{H}^{k}$ is modified in terms of Eqs. (19) and (20).

Step 3. Successive approximation is continued until $D^{k}$ in Eq. (18) satisfies Eq. (21).

Step 4. Regarding $\overrightarrow{\boldsymbol{u}}^{k}$ and $\boldsymbol{H}^{k}$ as the solution at the $(n+1)$ time step, we may proceed with the next step.

\section{Results and Review of Analysis}

\subsection{Flow in a square cavity}

According to the algorithm mentioned above, flow in a square cavity, which has been already investigated theoretically and experimentally, is analyzed. The computational scheme for high Reynolds number flow is also examined. At Reynolds number 10000 , the mesh system is $50 \times 50$ and the division becomes smaller and denser toward the wall to increase the resolution in space. Figure 2 shows the boundary conditions and Fig. 3 shows the mesh division of the present analysis. The computation deals with subsequent flow since $t=0$ when the upper wall begins to move impulsively to the right. In the initial condition, the value of the velocity and the Bernoulli function are deemed 0 throughout the flow domain. In Fig. 4, stream lines are depicted by the distribution of an instantaneous stream function from initial time $t=0$ to $t=45$. The time indicates nondimensional time. Figure 5 shows the finally converged solution at time $t=140$. As mentioned, this analysis well describes the transient state of the cavity flow. The transient flow is consider- ably complex. A vortex formed at the corner on the uppcr right in Fig. 5 approaches the center of the cavity with time; then the main vortex repeats its rotational motion around that center. With the motion of the main vortex, secondary vortices are generated at the lower right, upper left and lower left of the cavity, separating from and mixing with each other. Thus, it is believed that this analysis can express the transient state which could not be captured by other means : e.g., steady solution or visualization. In order to confirm the validity of the above results, they are compared with other analytical methods or experiments. The convergent solution shown in Fig.5 is in good agreement with the computational result by Ghia et al. ${ }^{(6)}$ The result of the modifled GSMAC has very good accuracy although this calculation was performed only with Ghia et al.'s 1/28 mesh number. In addition, the velocity distribution of the central section is in good agreement with recent experimental

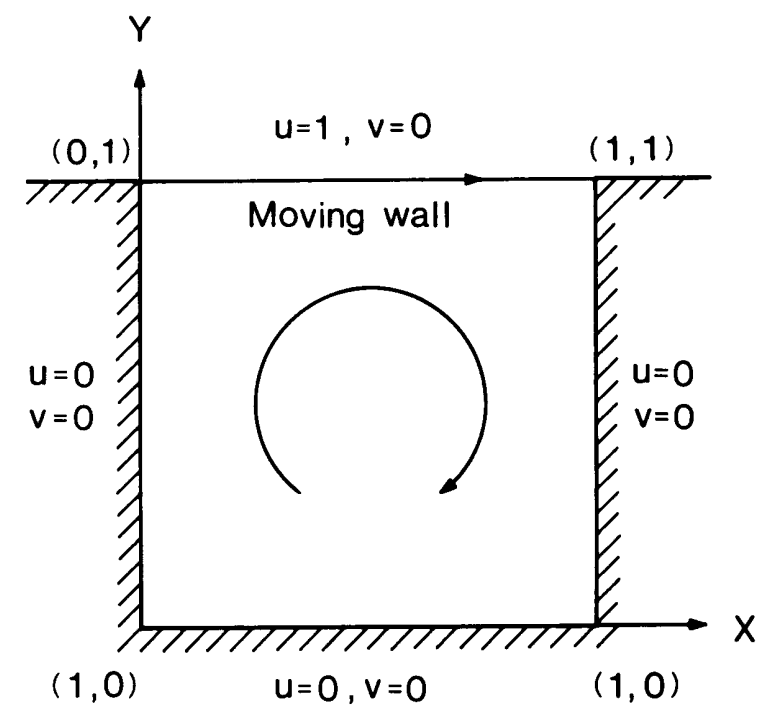

Fig. 2 Boundary conditions

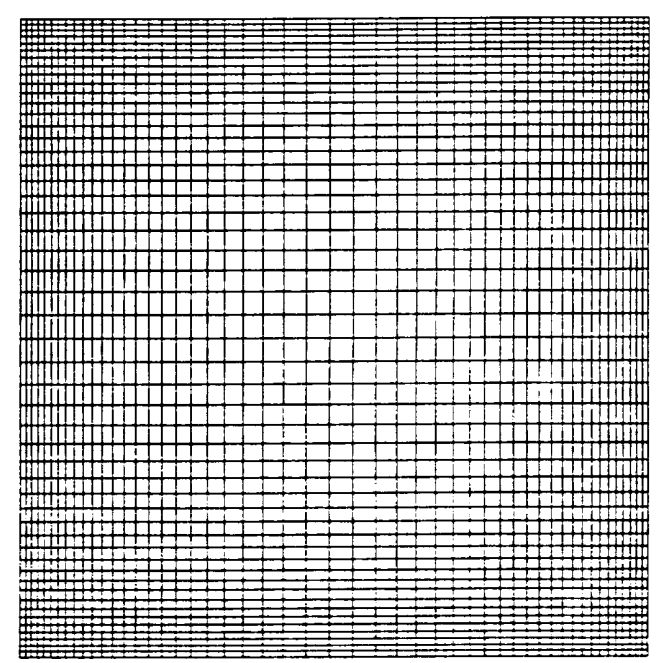

Fig. 3 Mesh used for the calculation 


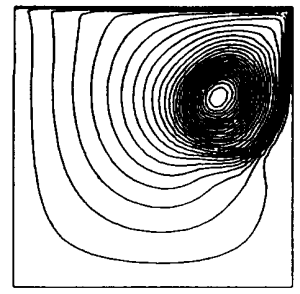

$t=10$

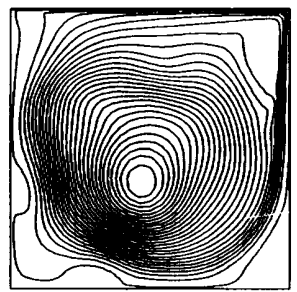

$t=30$

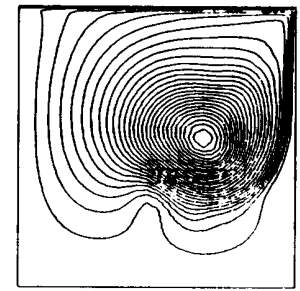

$t=15$

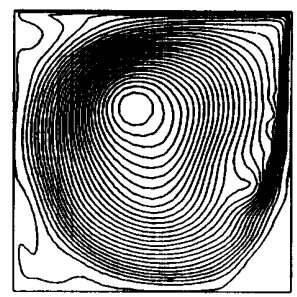

$t=35$

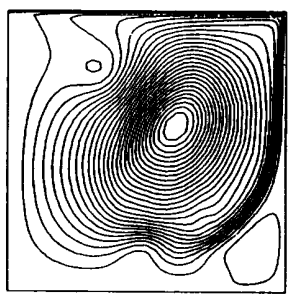

$t=20$

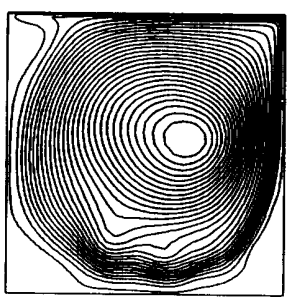

$\mathrm{t}=40$

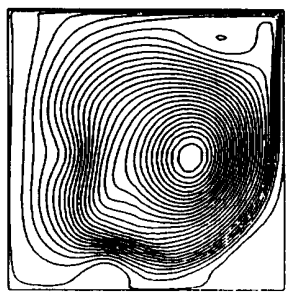

$t=25$

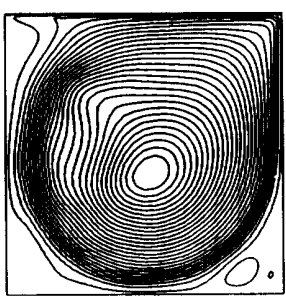

$\mathrm{t}=\mathbf{4 5}$

Fig. 4 Stream line in a square cavity $(R e=10000)$

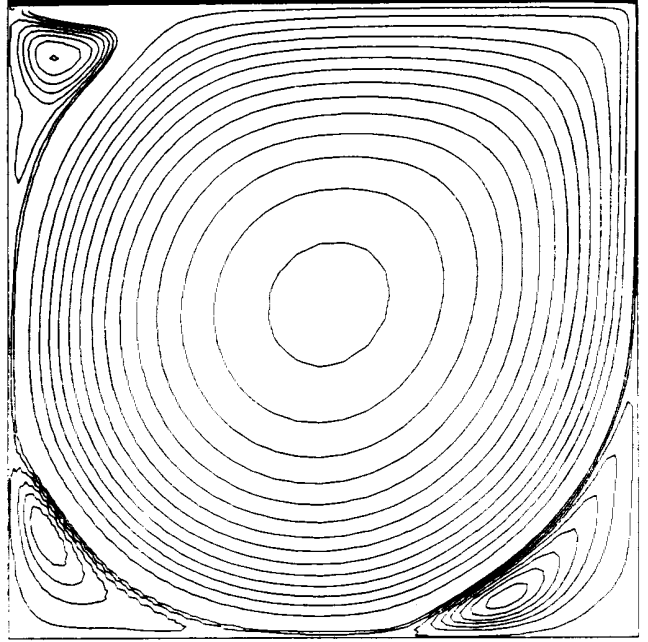

Fig. 5 Stream line in a square cavity ( $R e=10000$, convergent solution)

resuits obtained by Koseff and Street ${ }^{(7)}$. The aforesaid results also coincide with those of the ordinary GSMAC method $^{(3)(4)}$ [see Ref.(3) Table 2].

Even in the case for larger time interval $\Delta t$, the solution of the ordinary GSMAC method cannot converge; thus this modified GSMAC method is feasible. In the case of the same iteration number, this scheme' $\mathrm{s}$ convergence of the continuity equation in each cell is advanced by an order of $10^{-2}$ compared to the ordinary GSMAC method.

From the above-mentioned statement, we believe that the effectiveness of this scheme has been confirmed. This computation was performed on the HITAC S 810/20 (computer center, University of Tokyo ), requiring less than 1 hour to reach the final solution for all 28000 steps. This is very fast compared to an ordinary FEM and makes computation of an unsteady flow possible, which has hitherto not been treated due to lack of time.

\subsection{Flow in a turbulent-type clean room}

For an example application of the analysis to a turbulent-type clean room, a square model with one entrance and one exit is analyzed. The same mesh system with one square cavity is used. In the initial condition, velocity and $\boldsymbol{H}$ are deemed zero in all calculation regions. Iteration is carried out to satisfy the condition of the equation of continuity $\varepsilon=10^{-4}$. The initial values at $\boldsymbol{t}=0$ in this analysis are determined as a result of the iteration.

Figures $6 \sim 8$ show the velocity vector, stream lines and vorticity distribution, respectively, at identical times from $t=0$ to $t=50$. This clean room model is comparatively simple but the Reynolds number of the flow is 10000 . The internal flow in such conventional clean rooms is extremely complicated. It is reported ${ }^{(8)}$ that a vortex formed near the exit grows and exhibits cyclical behavior-formation, break up, integration and extinction-with a large circulatory motion. This analysis also shows a very complex flow pattern, that is, generation, mixing and extinction of a vortex; it successfully simulates the change in the unsteady motion of internal behavior around a large circulatory flow. Air blowing down from the entrance flows, without dissipation, near the exit. Air flowing along the bottom surface goes up along the side wall. The upstream impinges the downstream from the entrance and forms a large vortex. The upstream also occurs along the right-side wall toward the exit. Here, a formed vortex interferes with the downstream of air 
and eventually shows cyclical phenomena-formation, break up and extinction. On the left wall, which has no exit, a large vortex grows and begins a circulatory motion. Secondary vortices induced from such flow movements along the wall merge into a vortex situated in the upper left portion of the room. This means low ventilation efficiency. From the standpoint of purification efficiency and energy conservation, there are many ways of increasing efficiency. For example, another drainage hole may be installed in a region where circulatory motion occurs. The number and position of such holes may be changed. In this way, the modified GSMAC provides solutions at high Reynolds number without using turbulence models and without introducing the upstream method. Therefore this scheme is very useful and is a meaningful method of
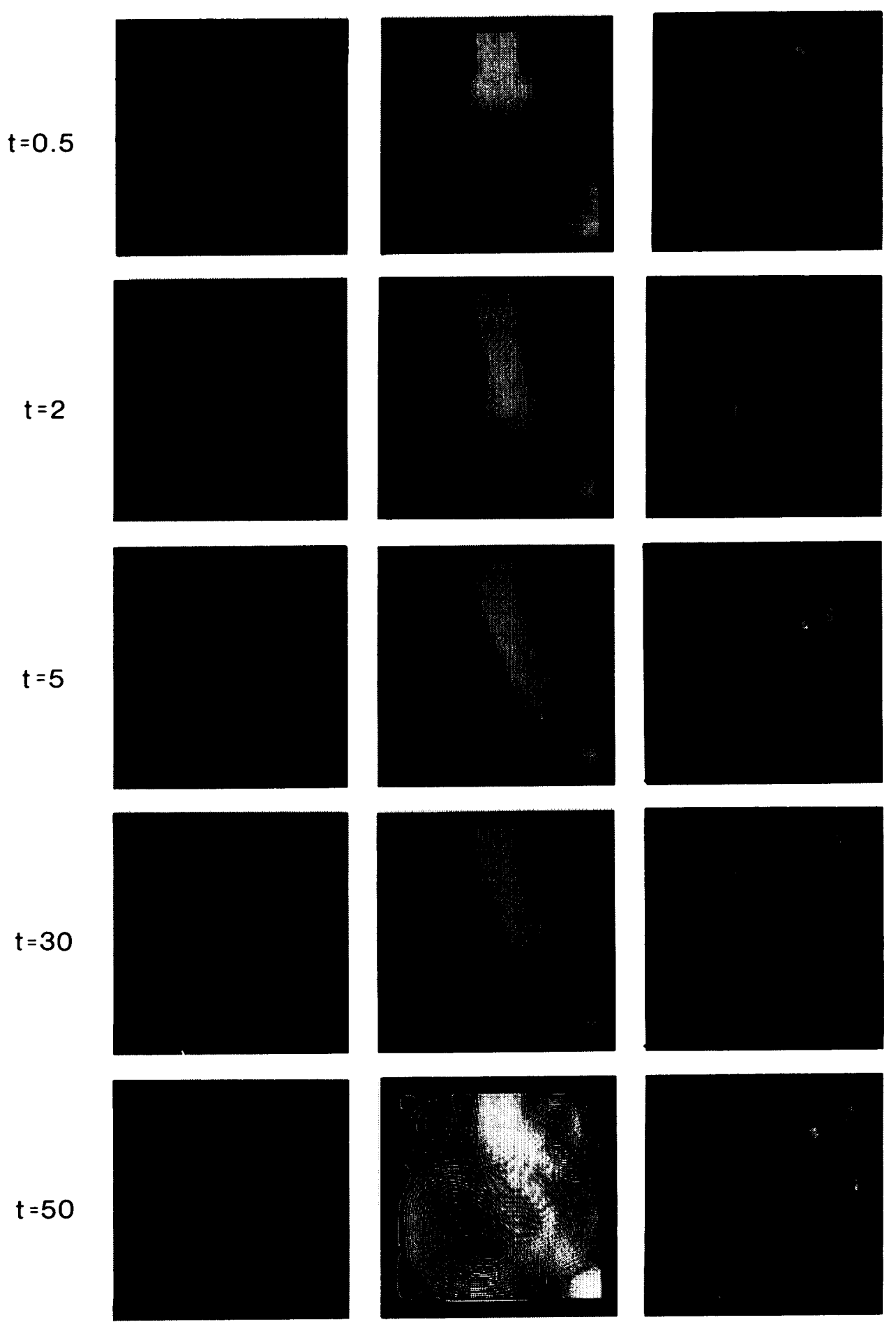

Fig. 6 Velocity vector $(\operatorname{Re}=10000)$

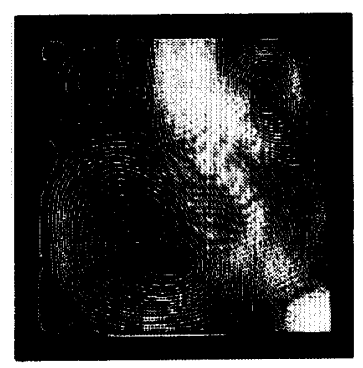

Fig. 7 Stream line $(R e=10000)$

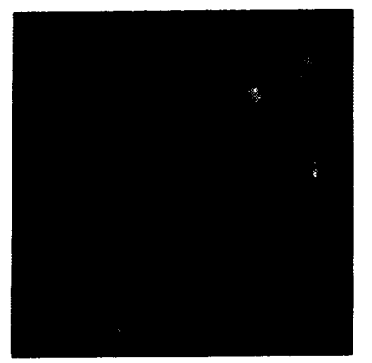

Fig. 8 Vorticity distribution $(\operatorname{Re}=10000)$ 
calculating the unsteady Navler-Stokes equation.

\section{Concluding Remarks}

This study deals with the verification of a computational scheme of the modified GSMAC method and tries to analyze transient air flow behavior in a turbulent-type clean room. The conclusions are as follows.

(1) To verify this scheme, the flow of Reynolds number 10000 in the lid-driven square cavity is analyzed, and the convergent solution is in good agreement with the results calculated by Ghia et al. The velocity distribution is compared with experimental results and is confirmed to be in good agreement. It also coincides with the previous result.

(2) The flow of Reynolds number 10000 in a square cavity is confirmed to show turbulent behavior. Certain phenomena are observed, such as the rotational motion of the main vortex around the center of gravity and transfer, and the mixing and extinction of secondary vortices near the wall.

(3) The transient behavior of flow in a turbulent-type clean room is analyzed. It is confirmed that large circulatory flow occurs in the room and that air flow near the entrance shows especially turbulent behavior.

(4) In this analysis, the computation has been shown to be stable and fast, compared to the ordinary FEM.

The above results show this method to be very effective.

\section{References}

(1) Kawal, H., Sawada, T. and Tanahashi, T., GSMAC-FEM for Incompressible Viscous Flow Analysis (A Modified GSMAC Method), Trans. Jpn. Soc. Mech. Eng., (in Japanese), Vol. 54, No. 507, B (1988), p. 3118.

(2) Cholin, A. J., On the Convergence of Discrete Approximations to the Navier-Stokes Equations, Math. Comput., Vol. 23 (1969), p. 341.

(3) Kanai, E. and Tanahashi, T., GSMAC - A New Finite Element Method for Unsteady Incompressible Viscous Flow Problems (1st Report, A Stable Method at High Reynolds Numbers), Trans. Jpn. Soc. Mech. Eng., (in Japanese), Vol. 53, No. 487, B (1987). p. 683.

(4) Tanahashi, T. and Saito, T., Kokyuroku-548, (in Japanese), (1985), p. 122, RIMS Kyoto Univ.

(5) Hirt, C. W., Nichols, B. D. and Romero, N. C., "SOLA" A Numerical Solution Algorithm for Transient Fluid Flows, LA -5852 (1975), LosAlamos Scientific Laboratories.

(6) Ghia, U., Ghia. K. N. and Shin, C. T., High-Re Solution for Incompressible Flow Using the Navier-Stokes Equations and a Multigrid Method, J. Comput. Phys., Vol. 48 (1982), p. 387.

( 7 ) Koseff, J. R. and Street, R. L., The Lid-Driven Cavity Flow : A Synthesis of Qualitative and Quantitative Observations, Trans. ASME, J. Fluid Eng., Vol. 106 (1984), p. 390.

(8) Murakami, S., Kato, S. and Chirifu, S., Visualization of Air Flow in Conventional Flow Type Clean Room with Laser Light Sheet, J. Flow Visualization Soc. Jpn., Vol. 6, No. 22 (1986), p. 387. 